\title{
AN ABSTRACT OSCILLATION THEOREM
}

\author{
KURT KREITH
}

Questions of oscillatory behavior are generally associated with Sturm-Liouville equations of the form

$$
-(d / d t)(p(t) d x / d t)+q(t) x=\mu x
$$

where (1) is singular at one or both ends of an interval $I=(a, b)$, $-\infty \leqq a<b \leqq \infty$. Numerous criteria exist, depending on the behavior of the coefficients $p(t)$ and $q(t)$, which assure that solutions of (1) are or are not oscillatory near a singular end point of $I$. However there also exist criteria for oscillatory behavior involving only the spectrum of the Sturm-Liouville operator

$$
\tau=-(d / d t)(p(t) d / d t)+q(t)
$$

in the Hilbert space $L_{2}(I)$ (see for example Dunford-Schwartz [1, Theorem XIII.7.40]). For lower semibounded operators these criteria state that if (1) is oscillatory then the essential spectrum of $\tau$ intersects $(-\infty, \mu]$, whereas if (1) is nonoscillatory, then the essential spectrum of $\tau$ does not intersect $(-\infty, \mu)$. These spectral criteria suggest that it may be of interest to generalize the notion of "oscillatory behavior" to solutions of certain operator equations of the form

$$
A x=\mu x
$$

where $A$ is an appropriate operator in a Hilbert space $\mathfrak{S}$ and $\mu$ is a real constant. The purpose of this paper is to examine one such generalization.

We shall assume throughout that $A$ is a symmetric operator which is bounded below in a Hilbert space $\mathfrak{S}$. By adding sufficiently large positive multiples of $x$ to both sides of (3), one may assume, without loss of generality, that $A$ satisfies

$$
(A x, x) \geqq\|x\|^{2}
$$

for all $x$ in $D_{A}$ (the domain of $A$ ). It will be useful to recall that a symmetric operator $A$ satisfying (4) has a selfadjoint extension $\bar{A}$, the Friedrichs extension, obtained as follows: complete $D_{A}$ under the norm $\|x \mid\|^{2}=(A x, x)$ to construct a Hilbert space $\mathfrak{T}$ with inner product $((\cdot, \cdot))$; define $D_{\bar{A}}$ to consist of those elements $y \in \mathfrak{F}$ for which there exists a sequence $x_{n}$ in $D_{A}$ such that

Received by the editors December 1, 1966. 


$$
\lim _{n \rightarrow \infty}\left\|y-x_{n}\right\|=0 \text { and } \lim _{m, \infty \rightarrow \infty}\left\|x_{m}-x_{n}\right\|=0 \text {. }
$$

Then, according to Friedrichs' theorem, there exists a unique selfadjoint extension $\bar{A}$ of $A$ for which $D_{\bar{A}} \subset \mathfrak{N}$ and $((x, y))=(x, A y)$ for all $x \in \mathfrak{M}$ and all $y \in \mathscr{D}_{\bar{A}}$. If $\tau$ is nonsingular on $I=(a, b)$ and $D_{\tau}$ is taken to be the class of infinitely differentiable functions with compact support on $I$, then $\bar{\tau}$ will be the selfadjoint extension of $\tau$ corresponding to the boundary conditions $x(a)=x(b)=0$.

Let $\mathfrak{S}_{k}$ be a closed linear subspace of $\mathfrak{S}_{\mathfrak{S}}$ for which $\mathscr{D}_{A} \cap \mathfrak{S}_{k}$ is dense in $\mathfrak{S}_{k}$ and let $P_{k}$ denote the projection operator with range $\mathfrak{S}_{k}$. In $\mathfrak{S}_{k}$ we define a symmetric operator $A_{k}$ as follows:

(i) $x \in \mathscr{D}_{A_{k}}$ if $x \in \mathscr{D}_{A}$ and $P_{k} x=x$,

(ii) if $x \in D_{A_{k}}$, then $A_{k} x=P_{k} A x$.

Since $A_{k} x \in \mathfrak{S}_{k}$ for all $x \in \mathscr{D}_{A_{k}}$, it is clear that $A_{k}$ is a symmetric operator in $\mathfrak{S}_{k}$, satisfies (4), and therefore has a Friedrichs extension, to be denoted by $\bar{A}_{k}$. Norms and inner products in $\mathfrak{S}_{k}$ will be denoted by a subscript $k$ outside the norm or inner product symbol.

Consider now the operator equation

$$
A^{*} x=\mu x,
$$

where $A^{*}$ is the adjoint of $A$.

Definition 1. We say that $\mathfrak{S}_{k}$ is a nodal domain for (5) if $A x$ $=P_{k} A x$ for all $x \in \mathfrak{S}_{k} \cap D_{A}$ and

$$
\inf _{x \in D_{\bar{A}_{k}}} \frac{\left(\bar{A}_{k} x, x\right)_{k}}{\|x\|_{k}^{2}}=\mu,
$$

where this infimum is achieved by an eigenfunction $u_{k} \in D_{\bar{A}_{k}}$.

Definition 2. We say that a solution $x_{0}$ of (5) is oscillatory if there exists a decomposition of $\mathfrak{S}$ into orthogonal closed subspaces.

$$
\mathfrak{S}=\sum_{k=0}^{\infty} \oplus \mathfrak{S}_{k}
$$

such that for $k \geqq 1$ each $\mathfrak{S}_{k}$ is a nodal domain for (5) and $P_{k} x_{0}$ is the required eigenfunction satisfying $\left(\bar{A}_{k} P_{k} x_{0}, P_{k} x_{0}\right)_{k}=\mu\left\|P_{k} x_{0}\right\|_{k}^{2}$. (The fact that we do not impose any conditions on $\mathfrak{S}_{0}$ corresponds to a lack of boundary conditions in (1).)

In one direction these definitions lead to spectral criteria for oscillatory behavior of (5) very similar to those which exist for (1).

THEOREM. If (5) has an infinite number of orthogonal nodal domains, then the essential spectrum of $\bar{A}$ intersects $[1, \mu] .^{1}$

${ }^{1}$ Since $(\bar{A} x, x) \geqq\|x\|^{2}$, we know that the spectrum of $\bar{A}$ cannot intersect $(-\infty, 1)$. 
Proof. We shall show that given any $\epsilon>0$ and any positive in teger $N$, there exists a set of $N$ linearly independent vectors $v_{1}, \cdots, v_{N}$ in $D_{A}$ such that for any nontrivial linear combination $v=c_{1} v_{1}+\ldots$ $+c_{N} v_{N}$, we have $(A v, v)<(\mu+\epsilon)\|v\|^{2}$. According to a principle adapted by Friedrichs [2], this implies either that $\bar{A}$ has at least $N$ eigenvalues in $[1, \mu+\epsilon)$ or else that the spectrum of $\bar{A}$ is not discrete below $\mu+\epsilon$; since $\epsilon$ is arbitrarily small, $N$ is arbitrarily large and $\bar{A}$ is bounded below, such a construction implies that the spectrum of $\bar{A}$ is not discrete in $[1, \mu]$.

To construct the set $v_{1}, \cdots, v_{N}$, let $u_{1}, \cdots, u_{N}$ denote the normalized eigenfunctions of $\bar{A}_{1}, \cdots, \bar{A}_{N}$ corresponding to the eigenvalue $\mu$. By the definition of $\bar{A}_{k}$, for every $u_{k}$ there exists a sequence $v_{k l}$ satisfying

(i) $v_{k l} \in D_{A} ; \quad k=1, \cdots, N ; \quad l=1,2, \cdots$;

(ii) $P_{k} v_{k l}=v_{k l} ; \quad k=1, \cdots, N ; \quad l=1,2, \cdots$;

(iii) $\lim _{l \rightarrow \infty}\left\|\mid P_{k} v_{k l}-u_{k}\right\|_{k}=0$;

(iv) $\lim _{l \rightarrow \infty}|| P_{k} v_{k l}-u_{k} \|_{k}=0$.

For $k=1, \cdots, N$ we have

$$
||\left|v_{k l}\right|||=\left.||\left|P_{k} v_{k l}\right|\right|_{k} \leqq||\left|P_{k} v_{k l}-u_{k}\right|||_{k}+\left|\left\|u_{k} \mid\right\|_{k}\right.
$$

and

$$
\text { | }\left|u_{k}\right|\left\|_{k}=\mu^{1 / 2}\right\| u_{k} \|_{k} \leqq \mu^{1 / 2}\left(\left\|u_{k}-P_{k} v_{k l}\right\|_{k}+\left\|v_{k l}\right\|\right) .
$$

Combining these inequalities

$$
\left\|v_{k l}\right\| \mid \leqq\left\|P_{k} v_{k l}-u_{k}\right\| \|_{k}+\mu^{1 / 2}\left(\left\|u_{k}-P_{k} v_{k l}\right\|_{k}+\left\|v_{k l}\right\|\right) .
$$

In light of (iii) and (iv) and the fact that $\lim _{l \rightarrow \infty}\left\|v_{k l}\right\|=1$, we can choose $l_{0}$ sufficiently large so that

$$
\left\|v_{k l_{0}} \mid\right\|<(\mu+\epsilon)^{1 / 2}\left\|v_{k l_{0}}\right\| ; \quad k=1,2, \cdots, N .
$$

Defining $v_{k}=v_{k l_{0}}$, and noting that

$$
\begin{gathered}
\left(v_{j}, v_{k}\right)=0 \quad \text { for } j \neq k ; \quad\left(A v_{j}, v_{k}\right)=0 \text { for } j \neq k ; \\
\left(A v_{k}, v_{k}\right)<(\mu+\epsilon)\left\|v_{k}\right\|^{2},
\end{gathered}
$$

it follows that for any $v=c_{1} v_{1}+\cdots+c_{N} v_{N} \neq 0$

$$
(A v, v)=\sum_{k=1}^{N}\left|c_{k}\right|^{2}\left(A v_{k}, v_{k}\right)<(\mu+\epsilon) \sum_{k=1}^{N}\left|c_{k}\right|{ }^{2}\left\|v_{k}\right\|^{2}=(\mu+\epsilon)\|v\| \|^{2},
$$

which completes the proof.

As an immediate consequence we have the following.

Corollary. If $A^{*} x=\mu x$ has an oscillatory solution, then the spectrum of $\bar{A}$ is not discrete in $[1, \mu]$. 
If the operator $A$ has finite deficiency indices, then the essential spectrum of all selfad oint extensions is the same, and this fact enables one to formulate criteria in terms of the essential spectrum of $A$ instead of $\bar{A}$. This remark applies in particular to the operator $\tau$ whose deficiency indices are at most 2.

For the operator $\tau$ we also have the following converse of Theorem 1. If (1) has a nonoscillatory solution, then the essential spectrum of $\tau$ does not intersect $[1, \mu)$. That such a converse does not hold in our more general setting is indicated by an elementary example.

Consider $A x \equiv-\partial^{2} x / \partial s^{2}-\partial^{2} x / \partial t^{2}+q(t) x$, where $q(t)$ is chosen such that the singular selfadjoint Sturm-Liouville system

$$
\begin{aligned}
-d^{2} y / d t^{2}+q y & =\lambda y ; \quad 0<t \leqq 1, \\
y(1) & =0,
\end{aligned}
$$

has a spectrum composed of an eigenvalue at $\lambda=-1$, some continuous spectrum in the interval $(-3,-2)$, a finite number of eigenvalues for $\lambda<-3$, and an arbitrary mixture of discrete and continuous spectrum for $\lambda>-1$. Defining $R=\{(s, t) \mid 0<s<\pi ; \quad 0<t<1\}$ and setting $\mathfrak{S}=L_{2}(R)$ we can solve the boundary value problem

$$
\begin{aligned}
A x=0 & \text { in } R, \\
x=0 & \text { on } \partial R \cap\{(s, t) \mid t>0\}
\end{aligned}
$$

by separation of variables. Denoting this boundary value problem by $A^{*} x=0$ and setting $x(s, t)=S(s) T(t)$, we get

$$
S(s)=\sin n s, \quad-T^{\prime \prime}+q T=-n^{2} T
$$

for $n=1,2, \cdots$. Setting $n=1$, it follows that $A^{*} x=0$ has a square integrable oscillatory solution; however, for $n>1$ no solution of $A^{*} x=0$ is oscillatory.

This example suggests that a satisfactory converse to Corollary 1 is likely to be elusive in any abstract setting general enough to include singular elliptic operators.

\section{BIBLIOGRAPHY}

1. N. Dunford and J. T. Schwartz, Linear operators, Part II, Interscience, New York, 1963.

2. K. O. Friedrichs, Criteria for discrete spectra, Comm. Pure Appl. Math. 3 (1950), 439-449.

University of California, Davis 